\title{
Objective Quantification of Opioid Usage After Thyroid Surgery
}

\author{
Andrew Agnew ${ }^{10}$ Sean Carroll ${ }^{2}$ Rick Fornelli ${ }^{3,4}$ Stephen Schell ${ }^{2}$ Kirk Steehler ${ }^{2}$ \\ ${ }^{1}$ Department of Medical Education, University of Pittsburgh Medical \\ Center (UPMC), Erie, PA, United States \\ 2Department of Otolaryngology Head and Neck Surgery, UPMC \\ Hamot, Erie, PA, United States \\ ${ }^{3}$ Department of Otolaryngology Head and Neck Surgery, West Virginia \\ University School of Medicine, Morgantown, WV, United States \\ ${ }^{4}$ Department of Otolaryngology, UPMC Hamot, Erie, PA, United States

\begin{abstract}
Address for correspondence Andrew Agnew, PhD, Department of Medical Education, University of Pittsburgh Medical Center, 201 State Street, Erie, Pennsylvania 16550, United States (e-mail: aw.agnew4@gmail.com).
\end{abstract} \\ Int Arch Otorhinolaryngol 2020;24(4):e482-e486.
}

\begin{abstract}
Keywords

- thyroidectomy

- opioid

- postoperative pain

- head and neck surgery

- otolaryngology
\end{abstract}

\section{Introduction}

It is well established that America is in the midst of an opioid crisis. Forty-six people die every day from overdoses involving prescription opioids. ${ }^{1,2}$ It has been shown that shortterm opioid use is associated with a higher likelihood of longterm misuse. An excess of opioid analgesic prescription is associated with diversion. ${ }^{3}$

Individuals misusing opioids often obtain them from family or friends, and opioids are now considered a gateway drug to heroin. ${ }^{4}$ Among new heroin users during 2000 to 2013, approximately 3 out of 4 report having misused prescription opioids prior to using heroin. ${ }^{3}$

There is wide variation in postoperative prescribing practices of otolaryngologists. ${ }^{5}$ Research has shown that softtissue surgery is considered less painful than any skeletal surgery. ${ }^{3}$ In the last 2 years, multiple articles have been published indicating that the amount of opioid pain medication needed after discharge from thyroid and parathyroid surgeries is low. ${ }^{6-10}$ While reaching a similar conclusion, our received

September 29, 2019

accepted

November 15, 2019
DOI https://doi.org/

10.1055/s-0039-3402496. ISSN 1809-9777.
Copyright (e 2020 by Thieme Revinter

Publicações Ltda, Rio de Janeiro, Brazil
License terms

()ㅇ (1) $\circledast$ 
study is the first to objectively examine the quantity of opioid pain medication consumed between postoperative discharge and office follow-up.

\section{Methods}

Patients undergoing partial, total, or complete thyroidectomy by four surgeons in our teaching otolaryngology practice from August 2017 to December 2018 were considered for our study. Appropriate Institutional Review Board approval was obtained (IRB\#17-02-01). Given the prospective and voluntary nature of our data collection, written informed consent was obtained from each research subject. Exclusion criteria included chronic neck pain, active opioid usage, age under 18 , pregnancy, or incarceration.

The Centers for Disease Control (CDC) has provided a calculator to standardize the morphine milligram equivalent (MME) across different varieties and strengths of pain medication $^{11}$ (- Table 1). Utilizing MME allows for easier communication of pain data across different pain medications. It is worth noting that $1 \mathrm{mg}$ of oxycodone corresponds to 1.5 MME and $1 \mathrm{mg}$ of hydrocodone corresponds to 1.0 MME.

The aim of the present study was to capture how many combination pills of $7.5 \mathrm{mg}$ of hydrocodone and $325 \mathrm{mg}$ of acetaminophen patients used after hospital discharge for up to 7 days. Prior to the study, our group's prescribing practices were based on physician preference and prior experience. To satisfy the IRB mandate that study participation would not limit access to pain medication postoperatively, we chose the upper range of prescribing practices at 30 pills (225 MME).

It is our current practice to keep patients overnight after all thyroid surgeries. On the day of discharge, consenting patients were enrolled in our study and given a log sheet to keep track of the number of $7.5 \mathrm{mg}$ hydrocodone $325 \mathrm{mg}$ acetaminophen combination pills they used per day at home. The log sheet included a copy of the validated Wong-Baker faces pain rating scale to rate their average pain for each day

Table 1 Centers for Disease Control (CDC) conversion chart for calculating morphine milligram equivalents (MME)

\begin{tabular}{|l|l|}
\hline $\begin{array}{l}\text { Opioid (doses in mg/day, except where } \\
\text { noted) }\end{array}$ & $\begin{array}{l}\text { Conversion } \\
\text { factor }\end{array}$ \\
\hline Codeine & 0.15 \\
\hline Fentanyl transdermal (in mcg/hr) & 2.4 \\
\hline Hydrocodone & 1 \\
\hline Hydromorphone & 4 \\
\hline Methadone & \\
\hline $1-20 \mathrm{mg} /$ day & 4 \\
\hline $21-40 \mathrm{mg} /$ day & 8 \\
\hline $41-60 \mathrm{mg} /$ day & 10 \\
\hline$\geq 61-80 \mathrm{mg} /$ day & 12 \\
\hline Morphine & 1 \\
\hline Oxycodone & 1.5 \\
\hline Oxymorphone & 3 \\
\hline
\end{tabular}

at home after discharge. Written permission was obtained from the Wong Baker Foundation for usage of the scale in our study. $^{12}$

Patients were instructed to bring their pill bottle to their first postoperative appointment, which was at approximately one week postoperatively. At the follow-up appointment, the log sheet was collected. The patient's remaining pills were counted in accordance with the IRB approved protocol and any remaining pills were returned to the patient. The inoffice pill counts added a measure of objectivity to our study and eliminated recall bias.

Patients were excluded if they failed to bring either the pill bottle or the log sheet to the first follow-up appointment to maintain accuracy of the pill counts. A total of $64 / 83$ (77\%) of the patients that originally consented to the study completed the log sheet and brought their pill bottle to the first follow-up appointment as instructed.

At the conclusion of the study, data was analyzed retrospectively utilizing the patient's medical record number from the log sheets to obtain relevant study information. Statistical analysis was performed using IBM SPSS Statistics for Windows version 24 (IBM Corp, Armonk, NY, USA) and included 2-tailed $t$-tests for comparison of continuous variables and linear regression analysis to evaluate potential relationships amongst the number of tablets and average pain per day, length of stay, age, gender, surgeon, hospital site, procedure, smoking status, number of previous surgeries, number of allergies, number of medical comorbidities, weight, and body mass index (BMI). Statistical significance threshold for 2-sided $p$-values was less than 0.01

\section{Results}

Sixty-four patients met the inclusion criteria, including returning to their first postoperative appointment with their pill bottle and pain log sheet. The mean age of the patients was 57.2 years, including 48 females (75\%). No patient experienced a postoperative complication that required return to the operating room. No patient requested a refill of pain medication at the follow-up appointment.

Patients were prescribed a standard regimen of $30 \mathrm{com}-$ bination pills of $7.5 \mathrm{mg}$ of hydrocodone and $325 \mathrm{mg}$ of acetaminophen (225 MME). Twenty-five (39\%) patients either did not fill their prescription or used zero pills. There was no difference in pill consumption between genders $(p=0.904)$, hospital at which the procedure took place $(p=0.550)$, nor smoking status $(p=0.506)$ ( - Table 2$)$.

Pain per day did correlate strongly with pills consumed per day. Patients in more pain subjectively consumed more pills objectively $(p<0.001)$ ( - Fig. 1 ). There was a statistically significant negative correlation between mean pills per day and age, indicating that older patients tend to take fewer pills $(\mathrm{r}-0.404, p=0.001)$.

There was no correlation with weight $(p=0.881)$, BMI $(p=0.521)$, length of hospital stay $(p=0.088)$, number of medication allergies ( $p=0.839)$, number of prior surgeries $(p=0.848)$, number of home medications $(0.278)$, nor number of medical comorbidities $(p=0.340)$. 
Table 2 Patient demographics that did not reach statistical significance

\begin{tabular}{|l|l|l|l|}
\hline & $\begin{array}{l}\text { Number of } \\
\text { patients }\end{array}$ & $\begin{array}{l}\text { Mean pills } \\
\text { per day }\end{array}$ & $p$-value \\
\hline Gender & & & \\
\hline Male & 16 & 0.7469 & \\
\hline Female & 48 & 0.7143 & 0.904 \\
\hline Hospital & & & \\
\hline A & 35 & 0.7998 & \\
\hline B & 29 & 0.6650 & 0.550 \\
\hline Smoking status & & & \\
\hline Former & 6 & 1.1548 & \\
\hline Never & 47 & 0.7096 & \\
\hline Current & 11 & 0.6364 & 0.506 \\
\hline Procedure & & & \\
\hline Hemithyroidectomy & 28 & 0.7824 & \\
\hline Total and complete & $32 ; 4$ & 0.7248 & 0.735 \\
\hline
\end{tabular}

\section{Discussion}

At the beginning of our study, there was a paucity of evidence available to guide prescribing patterns. The surgeons in our practice chose $7.5 / 325 \mathrm{mg}$ hydrocodone/acetaminophen pills over 5/325 mg hydrocodone/acetaminophen or oxycodone based on training and experience. Our data supports the trend amongst recent publications indicating that pain is relatively mild postoperatively after thyroid surgery for most patients. By utilizing pill counts, we were able to objectively capture data on pill consumption for the first time in the thyroid literature.

A common trend amongst published literature regarding the amount of opioid pain medication prescribed postoperatively is a reduction in the amount of opioid medication prescribed after analyzing patient consumption. ${ }^{6-10,13,14}$ We, likewise, found this to be true for our practice.

Therapeutic use of opioids places the patient at increased risk for opioid abuse. ${ }^{15}$ Overdose deaths involving prescription opioids were 5 times higher in 2017 than in 1999. Opioids were involved in 47,600 overdose deaths in 2017 ( $67.8 \%$ of all drug overdose deaths). ${ }^{2}$
Prescription opioids have been established as a gateway drug to heroin abuse. Moreover, there has been a rise in fentanyl abuse, overdose, and death with prior prescription opioid usage and heroin abuse as risk factors. ${ }^{4,16}$ In 2015 , a sharp increase in fentanyl drug confiscations and fentanylrelated overdose fatalities lead the CDC and Drug Enforcement Administration (DEA) to issue a nationwide health alert. ${ }^{17,18}$

Fortunately, there has been an increasing number of publications in recent years to guide thyroid surgeon prescribing practices. In a 2017 article by Lou et al, the authors surveyed 313 patients on opioid consumption after discharge from thyroid or parathyroid surgery at the first postoperative visit. The average visit was 11 days postoperatively and relied on accurate patient recall. In their study, the authors defined $1 \mathrm{MME}$ as equivalent to one tablet of hydrocodone/acetaminophen 5/325 mg. A total of 93\% of their patients had adequate pain control with 20 or fewer tablets. They recommended discharge of patents with 20 tablets of hydrocodone/acetaminophen $5 / 325 \mathrm{mg}$. Prior to the study, the authors averaged 30 tablets on discharge. In conventional MME, using the CDC calculator the authors went from 150.0 to 100.0 MME. $^{6}$

More recently, in 2018, a large cohort of 1,702 patients undergoing thyroid or parathyroid surgery was examined by Shindo et al In their study, they examined the MME each patient was discharged with and whether the patients called to request a refill for more opioid pain medication or not. They found that patients who were prescribed 75.0 MME or less did not call for a refill. An admitted limitation of the study by the authors was that they did not capture consumption of opioids at home. Again, after examining their prescribing practices, the authors decreased their average MME on discharge for parathyroidectomy (176.20-80.08 MME), hemithyroidectomy (204.65-102.31 MME), and total thyroidectomy (214.87102.29). An important point highlighted in the article was the value of a discussion in regards to postoperative pain expectations between physician and patient. Counseling alone can lead to lower opioid use postoperatively. ${ }^{7}$

In another 2018 study by Long et al, the authors reported the average number of opioid pills prescribed on discharge postoperatively to 237 thyroid and parathyroid surgery patients was 43.1 , with $84.4 \%$ receiving oxycodone/acetaminophen (323 MME). The authors also acknowledged that a limitation of their study was no data was captured

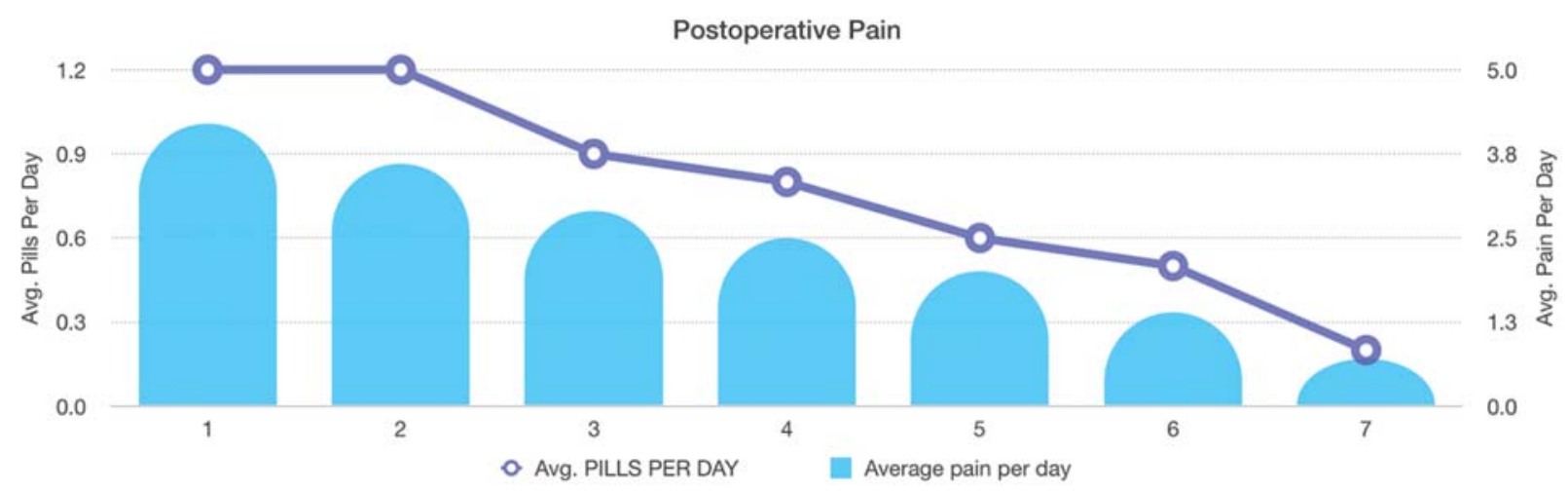

Fig. 1 Subjective pain scores correlated well with objective opioid pain medication consumption 
after hospital discharge and that after the study they have reduced their prescribing quantities. ${ }^{8}$

Another recent study published in 2018 by Tharakan et al showed that more than $80 \%$ of 209 patients undergoing total thyroidectomy, hemi thyroidectomy, and parathyroidectomy were able to have adequate pain control with 10 or fewer opioid pills. They conducted phone surveys and a variety of pain medications were prescribed on discharge. The majority of patients received 20 to 30 oxycodone 5-mg pills (150-225 MME). They found that older age was associated with lower pill consumption, and Charleston comorbidity index greater than 5 was associated with increased pill consumption. Again, telephone surveys were used, which introduces recall bias. $^{9}$

Perhaps, the most promising paper published in the last 2 years is by Militsakh et al in 2018, which showed the safety and effectiveness of multimodality anesthesia (MMA) for patients undergoing thyroid and parathyroid surgery. In their series of 528 patients spanning 3 calendar years, only 3 out of the 162 patients (1.9\%) enrolled in the MMA treatment pathway in 2017 required an opioid prescription on discharge. Briefly, patients received a combination of acetaminophen, ibuprofen, and gabapentin by mouth preoperatively. Postoperatively, patients were instructed to alternate acetaminophen and ibuprofen on a scheduled basis every 6 hours for the first 48 hours postoperatively. Only one hematoma was reported in the study cohort, and it was a patient involved in a motor vehicle accident as an unrestrained passenger. There were no reports of renal injury requiring unplanned hospital readmission. ${ }^{10}$

Our study was designed to objectively capture the amount of opioid pain medication consumed by patients at home after hospital discharge to determine an appropriate level of MME for discharge.

Limitations of our study include a possible Hawthorne effect, single practice study, lack of standardized anesthesia regimen, and exclusion of patients with an active opioid prescription. Although the anesthesia before, during, and immediately after the procedures was not standardized, it can be assumed that clinical standards of anesthesia were appropriately met. Furthermore, there was no difference in pain levels or pill consumption between the hospital cohorts. Long et al did note an increased level of pain and MME requirement for patients with an active opioid prescription while in the hospital. ${ }^{8}$ An area of future research would be to determine if this patient population requires more opioids. According to their published article, a cohort of such patients is being examined by Shindo et al currently. ${ }^{7}$

With increasingly restrictive laws requiring paper-only written prescriptions, surgeons must be cognizant of providing patients with enough pain medication to treat postoperative pain appropriately and maintain patient satisfaction, particularly patients that may live a great distance from the hospital or in another state.

Future areas of research would include but are not limited to continued postoperative pain research in other common head and neck procedures, a double-blinded study to inves- tigate if one opioid is more effect than another or versus nonopioid medication and the effects of multimodality anesthesia (MMA) on patients' opioids needs after discharge. Militsakh et al have provided strong evidence that opioid prescriptions may be avoidable with MMA. Their work also demonstrates safety of incorporating nonsteroidal antiinflammatory drugs (NSAIDs) into MMA before and after surgery without an increased bleeding risk. ${ }^{10}$

\section{Conclusion}

The present study objectively demonstrates that $85 \%$ of patients consumed fewer than 75.0 MME after thyroid surgery using in-office pill counts. Recent MMA research appears promising to dramatically reduce or even eliminate the need for opioid prescriptions upon postoperative discharge.

\section{Conflict of Interest}

The authors declare that there are no conflicts of interest.

\section{References}

1 Scholl L, Seth P, Kariisa M, Wilson N, Baldwin G. Drug and OpioidInvolved Overdose Deaths - United States, 2013-2017. MMWR Morb Mortal Wkly Rep 2018;67(5152):1419-1427

2 CDC WONDER [Internet]. Centers for Disease Control and Prevention. Centers for Disease Control and Prevention; [cited 2019Sep8]. Available from: http://wonder.cdc.gov/

3 Cicero TJ, Ellis MS, Surratt HL, Kurtz SP. The changing face of heroin use in the United States: a retrospective analysis of the past 50 years. JAMA Psychiatry 2014;71(07):821-826

4 Kelly MA. Addressing the Opioid Epidemic With Multimodal Pain Management. Am J Orthop 2016;45(07):S6-S8

5 Schwartz MA, Naples JG, Kuo C-L, Falcone TE. Opioid Prescribing Patterns among Otolaryngologists. Otolaryngol Head Neck Surg 2018;158(05):854-859

6 Lou I, Chennell TB, Schaefer SC, et al. Optimizing Outpatient Pain Management After Thyroid and Parathyroid Surgery: A TwoInstitution Experience. Ann Surg Oncol 2017;24(07):1951-1957

7 Shindo M, Lim J, Leon E, Moneta L, Li R, Quintanilla-Dieck L. Opioid Prescribing Practice and Needs in Thyroid and Parathyroid Surgery. JAMA Otolaryngol Head Neck Surg 2018;144(12):1098-1103

8 Long SM, Lumley CJ, Zeymo A, Davidson BJ. Prescription and Usage Pattern of Opioids after Thyroid and Parathyroid Surgery. Otolaryngol Head Neck Surg 2019;160(03):388-393

9 Militsakh O, Lydiatt W, Lydiatt D, et al. Development of Multimodal Analgesia Pathways in Outpatient Thyroid and Parathyroid Surgery and Association With Postoperative Opioid Prescription Patterns. JAMA Otolaryngol Head Neck Surg 2018;144(11): 1023-1029

10 Tharakan T, Jiang S, Fastenberg J, et al. Postoperative Pain Control and Opioid Usage Patterns among Patients Undergoing Thyroidectomy and Parathyroidectomy. Otolaryngol Head Neck Surg 2019;160(03):394-401

11 CDC Guideline for Prescribing Opioids for Chronic Pain | Drug Overdose I CDC Injury Center [Internet]. Centers for Disease Control and Prevention. Centers for Disease Control and Prevention; [cited 2019Sep8]. Available from: https://www.cdc.gov/ drugoverdose/pdf/calculating_total_daily_dose-a.pdf

12 Wong DL, Hackenberry-Eaton M, Wilson D, Winkelstein ML, Schwartz P. Wong's Essentials of Pediatric Nursing. 6th ed. St. Louis, MO: Mosby, 2001.

13 Locketz GD, Brant JD, Adappa ND, et al. Postoperative Opioid Use in Sinonasal Surgery. Otolaryngol Head Neck Surg 2019;160(03): 402-408 
14 Patel S, Sturm A, Bobian M, Svider PF, Zuliani G, Kridel R. Opioid Use by Patients After Rhinoplasty. JAMA Facial Plast Surg 2018;20 (01):24-30

15 Carroll I, Barelka P, Wang CKM, et al. A pilot cohort study of the determinants of longitudinal opioid use after surgery. Anesth Analg 2012;115(03):694-702

16 Madras BK. The President's Commission on Combating Drug Addiction and the Opioid Crisis: Origins and Recommendations. Clin Pharmacol Ther 2018;103(06):943-94
17 Archive HAN. - 00384|Health Alert Network (HAN) [Internet]. Centers for Disease Control and Prevention. Centers for Disease Control and Prevention; [cited 2019Sep8]. Available from: https://emergency.cdc.gov/han/han00384.asp

18 DEA Issues Nationwide Alert On Fentanyl As Threat To Health And Public Safety [Internet]. DEA. [cited 2019Sep8]. Available from: https://www.dea.gov/press-releases/2015/03/ 18/dea-issues-nationwide-alert-fentanyl-threat-health-andpublic-safety 